\section{Radiumtherapie wird geprüft}

Die amerikanische Arzneimittelbehörde FDA prüft die Zulassung von Radium-223-Dichlorid zur Behandlung des kastrationsresistenten Prostatakarzinoms mit Knochenmetastasen. Basis für den Zulassungsantrag bildet die Phase-III-Studie ALSYMPCA (ALpharadin in SYMptomatic Prostate CAncer), in der 921 Patienten mit kastrationsresistentem Prostatakarzinom und Knochenmetastasen im Abstand von vier Wochen bis zu sechs intravenöse Gaben von Radium-223 oder Placebo erhalten hatten. Das Ergebnis: Die Radium-223-Therapie verlängert das Gesamtüberleben der Patienten signifikant um 44\% (Hazard Ratio 0,695; p = 0,00007) [Parker C et al. J Clin Oncol. 2012;30(Suppl):Abstr. LBA4512]. Patienten, die mit Radium-223 und bestmöglicher Standardtherapie (BSoC) behandelt worden waren, überlebten durchschnittlich 3,6 Monate länger als diejenigen, die Placebo und BSoC erhalten hatten (14,9 vs. 11,3 Monate). red

Nach Informationen von Bayer Pharma

\section{Forschung zur Lebensqualität}

Am 7. Dezember 2012 wurden drei Wissenschaftler für herausragende Untersuchungen in der Onkologie und Lebensqualitätsforschung mit dem LillyQuality-of-Life-Preis 2012 ausgezeichnet. Jörg Heil aus Heidelberg erhielt den Preis für die Entwicklung der Breast Outcome Scale (BCTOS), eines Instruments zur Lebensqualitätsmessung bei Brustkrebs, das eine differenzierte Beuteilung des ästhetischen und funktionellen Ergebnisses bei einseitig brusterhaltend operierten Patientinnen erlaubt. Silke Schmidt und Holger Muehlan aus Greifswald entwickelten und validierten die Kurzversion eines bestehenden Instruments zur Messung der Lebensqualität bei chronisch kranken Kindern und Jugendlichen im Alter von 8-16 Jahren (DCGM-37). red

Nach Informationen von Lilly

Decitabin bei akuter myeloischer Leukämie

\title{
Schonende Therapie für ältere Patienten
}

\author{
Für ältere Patienten mit akuter \\ myeloischer Leukämie (AML) \\ kann Decitabin (Dacogen ${ }^{\circledR}$ ) eine \\ wirksame und gut verträgliche \\ Option sein.
}

Decitabin ist in Europa zur Therapie älterer Patienten ( $\geq 65$ Jahre) mit AML zugelassen, die nicht mehr für eine Standard-Induktionschemotherapie geeignet sind. Der Nachweis der Wirksamkeit, so Michael Lübbert, Freiburg, beruht auf der Phase-III-Studie DACO016, in der 485 über 65-jährige Patienten mit neu diagnostizierter AML eingeschlossen worden waren; sie erhielten randomisiert entweder niedrig dosiertes Decitabin $\left(20 \mathrm{mg} / \mathrm{m}^{2}\right.$ für 5 Tage alle 4 Wochen) oder wahlweise eine Supportivtherapie oder Cytarabin $\left(20 \mathrm{mg} / \mathrm{m}^{2}\right.$ für 10 Tage alle 4 Wochen) [Kantarjian HM et al. J Clin Oncol. 2012;30(21):2670-7]. Decitabin verlängerte das Gesamtüberleben signifikant um etwas mehr als die
Hälfte (median 7,7 vs. 5,0 Monate; Hazard Ratio 0,82; $\mathrm{p}=0,037$ ).

In einer deutschen Phase-II-Studie [Lübbert $M$ et al. Haematologica. EHA 2012] mit 227 Patienten konnte außerdem gezeigt werden, dass das Überleben umso länger ist, je besser der Allgemeinzustand ist, dass es nicht von der Zytogenetik beeinflusst wird, dass es bei hohen Leukozytenzahlen kürzer ausfällt und dass eine erfolgreiche Fortsetzung der Therapie nicht vom Auftreten einer kompletten Remission abhängt. Kasuistiken und Fallserien von bisher mehr als 170 Patienten belegen außerdem, dass sich die Behandlung mit Decitabin auch als Überbrückungsstrategie zu einer allogenen Stammzelltransplantation anbietet.

Josef Gulden

Satellitensymposium „Valuable Treatment Options for Older AML Patients" und Internationales Symposiums "Acute Leukemias XIV. Biology and Treatment Strategies", München, 25.02.2013; Veranstalter: Janssen-Cilag

Neue Daten zu Vemurafenib

\section{BRAF-Inhibition beim Melanom}

\section{Vemurafenib (Zelboraf ${ }^{\oplus}$ ) zeigt auch in der Langzeitbeobachtung gute Ergebnisse bei der Melanom- Behandlung. Die Kombination mit Cobimetinib könnte künftig eine weitere Therapieoption sein.}

Die auf dem ASCO-Jahreskongress 2012 vorgestellten Ergebnisse der Phase-III-Studie BRIM- 3 bestätigen den therapeutischen Vorteil von Vemurafenib gegenüber Dacarbazin bei bisher nicht behandelten Patienten mit nicht resezierbarem oder metastasiertem Melanom und einer BRAF-V600EMutation auch bei längerer Nachbeobachtung [Chapman PB et al. J Clin Oncol. 2012;30(Suppl): Abstr. 8502]. Die Auswertung ergab ein um fast vier Monate längeres medianes Gesamtüberleben bei Vemurafenib-Therapie (13,6 vs. 9,7 Monate, Hazard Ratio 0,$70 ; \mathrm{p}<0,001)$. Nach 12 Monaten lebten im Vemurafenib-Arm noch 56\% der Patienten, im Dacarbazin-Arm noch
44\%. Die Ergebnisse der Langzeitbeobachtung aus der Phase-I-Studie BRIM-1 zeigten mit einer 1-Jahres-Überlebensrate von $55 \%$ eine ähnliche Wirksamkeit, die 2-Jahres-Überlebensrate lag bei 35,6\%, die 3-Jahres-Überlebensrate bei $25,9 \%$, wobei $44 \%$ der Patienten auch nach Progress den BRAF-Inhibitor weiter erhielten.

Erste Untersuchungen zur Kombination von Vemurafenib mit dem MEK-Inhibitor Cobimetinib zeigten v. a. bei Vemurafenib-naiven Patienten eine ermutigende Wirksamkeit. In der auf dem ESMO 2012 vorgestellten Phase-Ib-Dosierungsstudie erhielten die Patienten 2-mal täglich $960 \mathrm{mg}$ Vemurafenib und über 21 Tage eines 28-tägigen Behandlungszyklus zusätzlich $60 \mathrm{mg}$ Cobimetinib [Gonzales $R$ et al. ESMO 2012; LBA 28]. In dieser Dosierung war die Kombinationstherapie sicher und verträglich möglich.

red

Nach Informationen von Roche 